This item is the archived peer-reviewed author-version of:

Colloidal transport through trap arrays controlled by active microswimmers

\title{
Reference:
}

Yang Wen, Misko Vyacheslav, Marchesoni Fabio, Nori Franco.- Colloidal transport through trap arrays controlled by active microsw immers Journal of physics : condensed matter - ISSN 0953-8984 - 30:26(2018), 264004

Full text (Publisher's DOI): https://doi.org/10.1088/1361-648X/AAC61B

To cite this reference: https://hdl.handle.net/10067/1519460151162165141 
ACCEPTED MANUSCRIPT

\section{Colloidal transport through trap arrays controlled by active microswimmers}

To cite this article before publication: Wen Yang et al $2018 \mathrm{~J}$. Phys.: Condens. Matter in press https://doi.org/10.1088/1361-648X/aac61b

\section{Manuscript version: Accepted Manuscript}

Accepted Manuscript is "the version of the article accepted for publication including all changes made as a result of the peer review process, and which may also include the addition to the article by IOP Publishing of a header, an article ID, a cover sheet and/or an 'Accepted Manuscript' watermark, but excluding any other editing, typesetting or other changes made by IOP Publishing and/or its licensors"

This Accepted Manuscript is @ 2018 IOP Publishing Ltd.

During the embargo period (the 12 month period from the publication of the Version of Record of this article), the Accepted Manuscript is fully protected by copyright and cannot be reused or reposted elsewhere.

As the Version of Record of this article is going to be / has been published on a subscription basis, this Accepted Manuscript is available for reuse under a CC BY-NC-ND 3.0 licence after the 12 month embargo period.

After the embargo period, everyone is permitted to use copy and redistribute this article for non-commercial purposes only, provided that they adhere to all the terms of the licence https://creativecommons.org/licences/by-nc-nd/3.0

Although reasonable endeavours have been taken to obtain all necessary permissions from third parties to include their copyrighted content within this article, their full citation and copyright line may not be present in this Accepted Manuscript version. Before using any content from this article, please refer to the Version of Record on IOPscience once published for full citation and copyright details, as permissions will likely be required. All third party content is fully copyright protected, unless specifically stated otherwise in the figure caption in the Version of Record.

View the article online for updates and enhancements. 


\title{
Colloidal transport through trap arrays controlled by active microswimmers
}

\author{
Wen Yang ${ }^{1}$, Vyacheslav R. Misko ${ }^{2,3}$, Fabio Marchesoni ${ }^{3,4}$, and Franco Nori ${ }^{3,5}$ \\ ${ }^{1}$ College of Materials Science and Engineering, Taiyuan University of Science and Technology, Taiyuan 030024, P. R. China \\ 2 TQC, Universiteit Antwerpen, Universiteitsplein 1, B-2610 Antwerpen, Belgium \\ 3 RIKEN, Wako-shi, Saitama 351-0198, Japan \\ 4 Dipartimento di Fisica, Università di Camerino, I-62032 Camerino, Italy and \\ 5 Physics Department, University of Michigan, Ann Arbor, MI 48109-1040, USA
}

(Dated: May 13, 2018)

\begin{abstract}
We investigate the dynamics of a binary mixture consisting of active and passive colloidal particles diffusing in a two-dimensional array of truncated harmonic wells, or traps. We explore the possibility of using a small fraction of active particles to manipulate a much larger fraction of passive particles, for instance, to confine them in or extract them from the traps. The results of our study have potential application in biology and medical sciences, for example, to remove dead cells or undesired contaminants from biological systems by means of self-propelled nano-robots.
\end{abstract}

PACS numbers:

\section{INTRODUCTION}

The capture and confinement of passive colloidal particles inside designated areas has been the focus of recent investigations aimed at new protocols for smart drug delivery and targeted cancer therapy [1, 2]. More generally, the study of active self-propelled particles, also called artificial microswimmers, has come to the forefront of biomedical research during the last decade [37]. Active particle systems mainly relate to assemblies of either microorganisms, like bacteria [8-12], or artificial self-phoretic colloids, epitomized by the so-called Janus particles [13-17]. Recent advances in the fabrication of active microswimmers suggest new techniques to control diffusion and transport of passive particles in confined geometries. For instance, pumping of passive species by active Janus particles has been demonstrated in asymmetric ratchet channels [18]. Extensive numerical simulations show that in such channels active Janus particles get rectified even in the presence of passive particles and, most remarkably, a tiny fraction of them is capable of dragging along most of the passive species, an effect called "autonomous pumping" [18]. Recently, persistent dragging of passive colloidal clusters by a single active Janus microswimmer has been observed experimentally even in smooth channels [19].

There have been experimental and theoretical efforts to describe the dynamics of colloidal particles immersed in an active fluid. Most of the reported work addressed the diffusion of colloids in swimming suspensions [20-23] or a passive tracer in a bacteria bath [24-27]. Other studies focused on turbulence in binary mixtures of active and passive particles $[28,29]$ and the effective interactions between them [30,31]. While the literature on such binary systems grew drastically in recent years, some basic questions concerning their equilibrium and non-equilibrium statistics remained unaswered.

Recently, the experiments of Koumakis and coworkers [2] demonstrated the targeted delivery of passive colloids by swimming bacteria. These authors designed spe- cific three-dimensional microstructures to serve as targeted area, where bacteria efficiently store the colloidal particles. Inspired by these observations, we simulated a model binary mixture of active Janus swimmers and passive particles suspended in a confined geometry, like a channel dotted with potential traps. For simplicity, the traps were represented by truncated parabolic potential wells, which closely mimic, for instance, the binding action of actual optical tweezers. We studied the interaction of active and passive species with the purpose of controlling colloidal clustering and transport by means of active microswimmers. In particular, we are interested in devising methods for delivering to and removing passive colloids from a targeted area by fine-tuning the concentration and the self-propulsion parameters of the active microswimmers.

The present paper is organized as follows. Our model and numerical approach are outlined in Sec. II. The accumulation of particles of mono-species systems within a parabolic trap is reported in Sec. III A. Next, in Sec. IIIB we investigate how effectively the active particles push the passive particles inside the trap. The diffusion of the active particles through trap arrays is simulated in Sec. III C. Finally, in Sec. III D we explore the possibility of using active microswimmers to empty the traps from the passive particles stored there. A few concluding remarks are drawn in the final Sec. IV.

\section{MODEL AND SIMULATION}

In our simulations, $N_{a}$ active (or self-propelled) particles and $N_{p}$ passive (or regular colloidal) particles move in a two-dimensional (2D) $L \times L$ box with a parabolic potential well of radius $r_{D}$ at its center. Periodic boundary conditions are imposed on the box sides. We model both active and passive particles as soft disks of equal radius, $r_{d}$, interacting pairwise through a repulsive short-range force. As reported in Refs. [18, 32], a particle of coordinates $\mathbf{r}_{i}=\left(x_{i}, y_{i}\right)$ is subject to an overdamped dynamics 
with Langevin equation (LE)

$$
\frac{\partial \mathbf{r}_{i}}{\partial t}=v_{0} \hat{\boldsymbol{\nu}}_{i}+\mu\left(\sum_{j \neq i} \mathbf{F}_{i j}+\mathbf{F}_{i}^{p}\right)+\boldsymbol{\eta}_{i}^{T}(t),
$$

where the self-propulsion speed, $v_{0}$, is positive only for the active particles, while the mobility, $\mu$, is the same for all constituents of the mixture. Here, $\mathbf{F}_{i j}$ is the repulsive interaction between particles $i$ and $j$, with $j \neq i$ denoting particles of either species. The particle repulsion force is defined as $\mathbf{F}_{i j}=\kappa \alpha_{i j} \hat{\mathbf{r}}_{i j}$, if $\alpha_{i j}=d-r_{i j}>0$ and $\mathbf{F}_{i j}=0$, otherwise. Here, $d=2 r_{d}$ denotes the particle' diameter, $\alpha_{i j}$ measures the overlap between particles $i$ and $j, r_{i j}$ being the distance between their centers, and, finally, $\kappa$ is a pair-independent stiffness constant. The attractive force exerted by the trap on particle $i$ is $\mathbf{F}_{i}^{p}=-A \mathbf{r}_{i}$, if $r_{i}<r_{D}$ and $\mathbf{F}_{i}^{p}=0$, otherwise. This corresponds to modeling the trap as a truncated harmonic potential well of strength $A$ and radius $r_{D}$ [33]. The unit vector $\hat{\boldsymbol{\nu}}_{i}=\left(\cos \theta_{i}, \sin \theta_{i}\right)$ represents the direction of the selfpropulsion velocity of the active particle of index $i$, the random direction of which, $\theta_{i}(t)$, is modeled as a Wiener process,

$$
\frac{\partial \theta_{i}}{\partial t}=\eta_{i}(t)
$$

driven by the Gaussian, zero mean-valued, local noise, $\eta_{i}(t)$, with autocorrelation function

$$
<\eta_{i}(t) \eta_{j}\left(t^{\prime}\right)>=2 D_{r} \delta_{i j} \delta\left(t-t^{\prime}\right)
$$

Note that the rotational diffusion coefficient, $D_{r}$, coincides with the reciprocal of the persistence time, $\tau_{r}=$ $1 / D_{r}$, of the exponentially time-correlated Brownian motion executed by a purely active particle [18]. However here we also consider the effects of thermal fluctuations, represented in Eq. (1) by the additive Gaussian noise $\boldsymbol{\eta}_{i}^{T}(t)$, with correlation functions,

$$
<\eta_{i \alpha}^{T}(t)>=0,<\eta_{i \alpha}^{T}(t) \eta_{j \beta}^{T}\left(t^{\prime}\right)>=2 D_{T} \delta_{i j} \delta_{\alpha \beta} \delta\left(t-t^{\prime}\right),
$$

where the indices $\alpha=1,2$ and $\beta=1,2$ denote the Cartesian coordinates $x$ and $y$, respectively, and $D_{T}$ is the thermal diffusion coefficient of the suspension.

Furthermore, in order to work with dimensionless quantities, we adopted the particle diameter, $d$, as the unit of length and the trap relaxation time, $(\mu \kappa)^{-1}$, as the unit of time. Fixed model parameters used in the simulation analysis presented next are $L=100, r_{D}=15, d=1$, $A=0.1, \kappa=10, \mu=1, D_{r}=0.005$, and $D_{T}=0.001$. The integration scheme of the model LE's is the same as in Ref. [18] with total running time $t_{e}=6 \times 10^{4}$ and integration time-step $\Delta t=0.001$. In all runs the mixture was initially prepared by generating a uniform random distribution for the positions of the particles and the orientations of the active ones.

In order to emphasize the control action exerted by the active particles on the dynamics of the mixture overall, we kept the levels of the angular, $D_{r}$, and thermal noise, $D_{T}$, relatively low. Of course, we ran extensive preliminary numerical tests for a variety of model parameters; the simulation results illustrated below are meant to serve as a proof of concept. For the same reason and to avoid unnecessary complications, we neglected hydrodynamic effects, which are known to contribute to the colloidal pair interaction, especially in 2D [34-36]. Finally, we mention that a similar model, also involving truncated parabolic traps, has already been investigated to study the statistics of a single species of trapped active Janus particles in the long-time ("thermodynamic") limit [33].

\section{SIMULATION RESULTS}

The system under consideration has two distinct relaxation timescales, one for the active dynamics, $\tau_{a}$, and one, $\tau_{p}$, associated with the thermal fluctuations. Both times depend on the trap parameters. The active Brownian motion outside the traps is characterized by the diffusion constant $D_{s}=v_{0}^{2} / 2 D_{r}$, which, in our simulation, is much larger than the thermal diffusion $D_{T}$. Accordingly, $\tau_{p}$ turns out to be orders of magnitude larger than $\tau_{a}$. Under experimental conditions, e.g., in biological systems, $\tau_{a}$ is typically of the order of seconds or minutes, while $\tau_{p}$ can be easily set of the order of hours, days, or even longer. This means that in our discussion $\tau_{p}$ will be regarded as infinitely long. Therefore, we simulated the relaxation dynamics of the binary mixture only up to long-lived quasi-stationary states; that is, states that are stationary on the time scale $\tau_{a}$. The asymptotic time relaxation toward thermal equilibrium can be numerically inyestigated only at higher mixture's temperatures, i.e., for values of $D_{T}$ corresponding to numerically accessible (shorter) relaxation times of the trapped passive particles, $\tau_{p}$.

\section{A. Trapping of a single species}

In this section we consider suspensions of only active or passive particles in the presence of a trap. In Fig. 1 (a) we plot the number of particles sitting in the trap, $N^{(t)}$ versus the total number of particles of either sort, $N$, active (red circles) or passive (black squares). We stress that the temperature was taken quite low, such that thermal diffusion is weak, $D_{T}=0.001$, and the condition $\tau_{p} \gg \tau_{a}$ holds. Accordingly, as anticipated above, these results and all system snapshots displayed in panels (b)-(e) of Fig. 1 have been obtained after simulation times, $t_{e}$, obeying the condition $\tau_{a}<t_{e}<\tau_{p}$.

As apparent in Fig. 1(a), for $N_{a}<1000$ all active particles enter the trap, which results in the linear branch of the curve $N_{a}^{(t)}$ versus $N_{a}$ with slope equal to one. For 

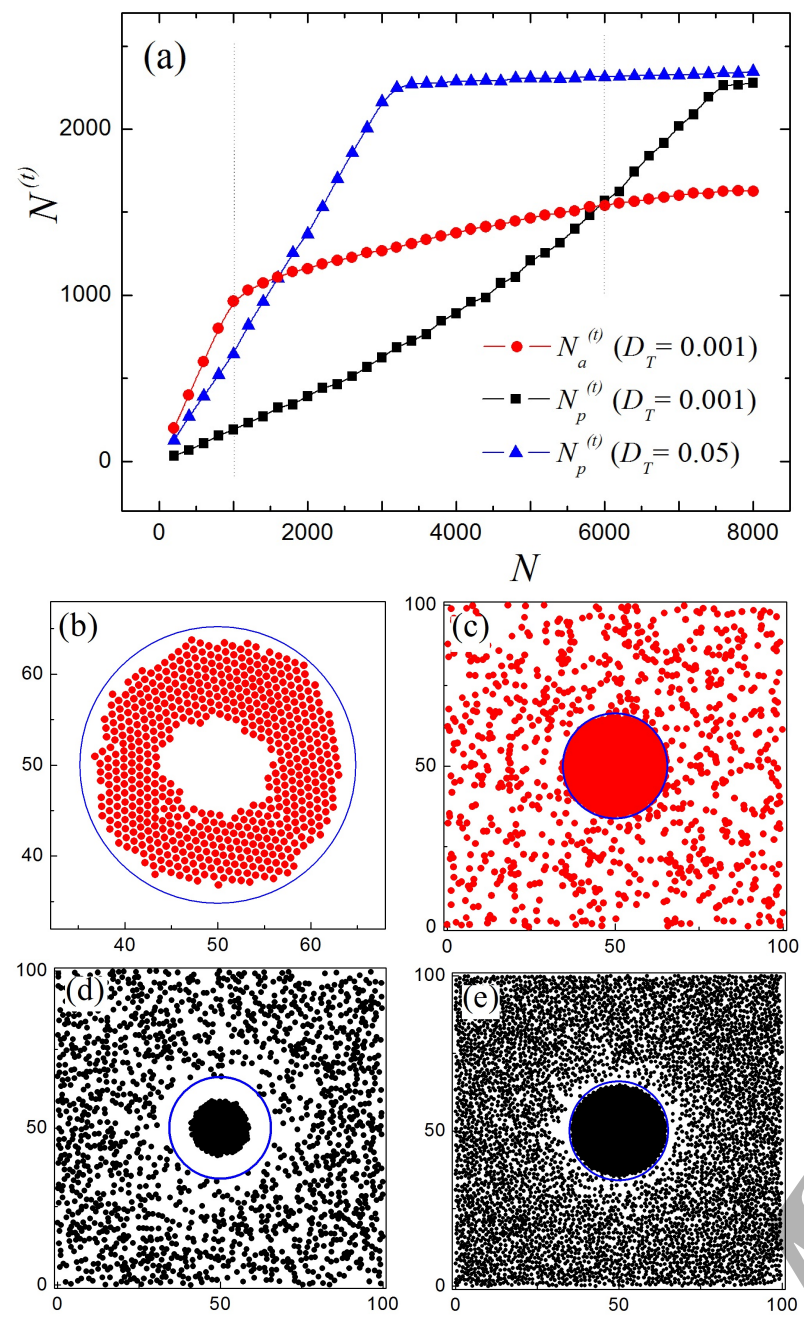

FIG. 1: (Color online). (a) Number of trapped particles $N^{(t)}$, versus total number of particles, $N$, for one-species suspensions of passive (squares and triangle) or active particles with $v_{0}=1.0$ (circles). Panels (b-e) are snapshots of different systems: (b) $N=N_{a}=600$; (c) $N=N_{a}=2000$; (d) $N=N_{p}=2000 ;$ (e) $N=N_{p}=7000$. The circles at the center of the simulation box delimit the parabolic trap. If not specified otherwise, the remaining simulation parameters are $L=100, r_{D}=15, d=1, A=0.1, \kappa=10, \mu=1$, $D_{r}=0.005, D_{T}=0.001$ and $t_{e}=6 \cdot 10^{4}$. The data set represented by triangles in (a) have been obtained for $D_{T}^{\star}=50 D_{T}$ and simulation time $t_{e}^{\star}=t_{e} / 3$.

$N_{a}>1000$, the $N_{a}^{(t)}$ curve develops a sub-linear behavior which finally levels off for asymptotically large $N_{a}$. In contrast, on the same timescale passive particles are uniformly distributed in the box and only a small fraction of them enters the trap. In other words, the spatial diffusion for active particles, Figs. 1(b, c), is much faster than for passive particles, Figs. 1(d, e). The sort of "halo" surrounding the traps in Figs. 1(d, e) is due to the slow thermal diffusion of the uniformly distributed passive particles, which over the run time length, $t_{e}$, fall inside the trap, thus generating a circular depletion re- gion around it.

These distinct relaxation transient properties play a crucial role in the dynamics of binary mixtures discussed in the forthcoming sections. In order to access the asymptotic behaviour, i.e., the thermodynamic equilibrium limit, one should considerably increase either the simulation time or the temperature. In Fig. 1(a) we plot, as an example, the curve $N_{p}^{(t)}$ versus $N_{p}$ for a much larger thermal diffusion, $D_{T}^{\star}=50 D_{T}$, but using a shorter simulation time, $t_{e}^{\star}=t_{e} / 3$. Under such conditions, the passive particle suspension behaves similarly to the active suspension described above, namely, exhibits a linear growing branch corresponding to the trapping of all passive particles for $N_{p}<3000$ and an asymptotic saturation for much larger $N_{p}$. However, as an interesting difference, one notices that here the horizontal asymptote is relatively higher and sets in immediately around $N_{p}=3000$, that is after the trap has been filled up. This is an obvious effect of the self-propulsion mechanism, which eventually hinders the containment of the active particles inside the trap.

B. Trapping of passive particles assisted by Janus microswimmers

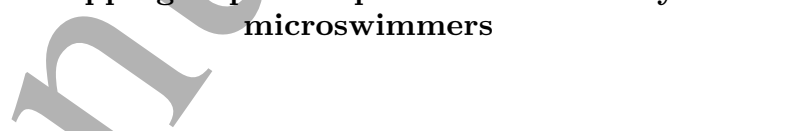

We inject now an increasing number of Janus particles, $N_{a}$, into a suspension containing a fixed number of passive particles, $N_{p}=1000$. In Fig. 2(a) we plot the number of trapped passive particles, $N_{p}^{(t)}$, versus $N_{a}$, for increasing values of the self-propulsion speed of the active microswimmers. The number of Janus particles trapped at the same time, $N^{(t)}$, is displayed in Fig. 2(b) for comparison.

A few properties of the mixture transient dynamics are apparent. First of all, trapping of passive particles is clearly expedited by the presence of active particles and grows more efficient upon increasing the concentration of the active component in the mixture.

More interesting is the dependence of the curves $N_{p}^{(t)}$ versus $N_{a}$ of Fig. 2(a) on the speed of the active Janus swimmers, $v_{0} . N_{p}^{(t)}$ first grows linearly (and slowly) with $N_{a}$ up to certain critical value, $N_{a}^{\star}$, and then bends upward toward its saturation value, $N_{p} \simeq 1000$. Such a sudden jump in the curves' profile shifts towards lower $N_{a}$ with increasing $v_{0}$, until the linear branch disappears altogether for $v_{0}>1.5$. All curves in Fig. 2(a) exhibit a small bump at about $N_{a}=600$, which gets more prominent as $N_{a}^{\star}$ approaches zero. The origin of these bumps is related with the formation of shells of active particles inside the trap (see snapshots in Fig. 3 for $N_{a}=500$ and 600$)$. For $N_{a} \leq 600$, the filling of the trap with active particles is proportional to $N_{a}$ [with slope close to 1 for $v_{0}$ up to 1.3, see Fig. 2(b)]. Such "quasi-stationary" shells encage passive particles inside the trap. The number of active particles in the shells saturates at about

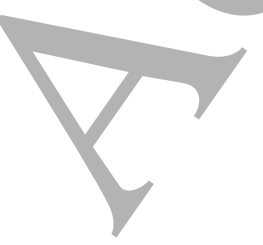



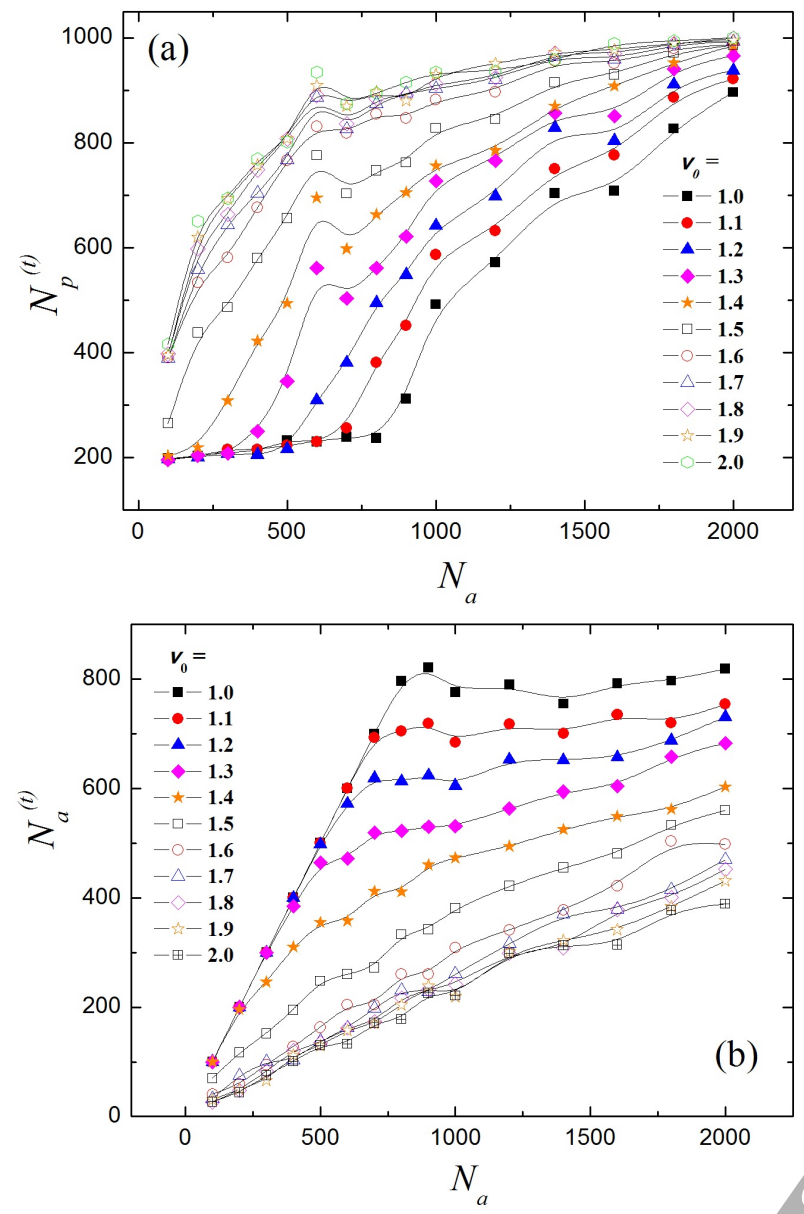

FIG. 2: (Color online). (a) Number of trapped passive particles in the trap $N_{p}^{(t)}$ versus total active particle number, $N_{a}$, for $N_{p}=1000$ and different $v_{0}$ (see legend). (b) The corresponding number of active particles, $N_{a}^{(t)}$, trapped at the same time. Note that the simulation data are represented by symbols, while the smooth interpolating curves are just a guide to the eyes. If not indicated otherwise, all simulation parameters are as in Fig. 1.

$N_{a}^{(t)} \approx 600$, as shown by the curves of Fig. 2(b) for $v_{0}=1.3-1.5$. The observed bumps in Fig. 2(a) correspond to these plateaus. Their appearance means that additional active particles do not contribute to the active shells surrounding the passive particles in the trap. These additional active particles thus freely move in and out of the trap, which eases the escape of the passive particles.

Moreover, one notices that for $v_{0}=2.0$ and $N_{a} \approx$ 600 about $95 \%$ of the passive particles become trapped, whereas in the absence of active swimmers only slightly more than $20 \%$ of them are found in the trap. This validates our starting idea of using active swimmers to control the dynamics of passive colloids: Active swimmers do efficiently help trap passive particles; they can be employed to clear up a working area from undesired stray particles by storing them in targeted areas, represented

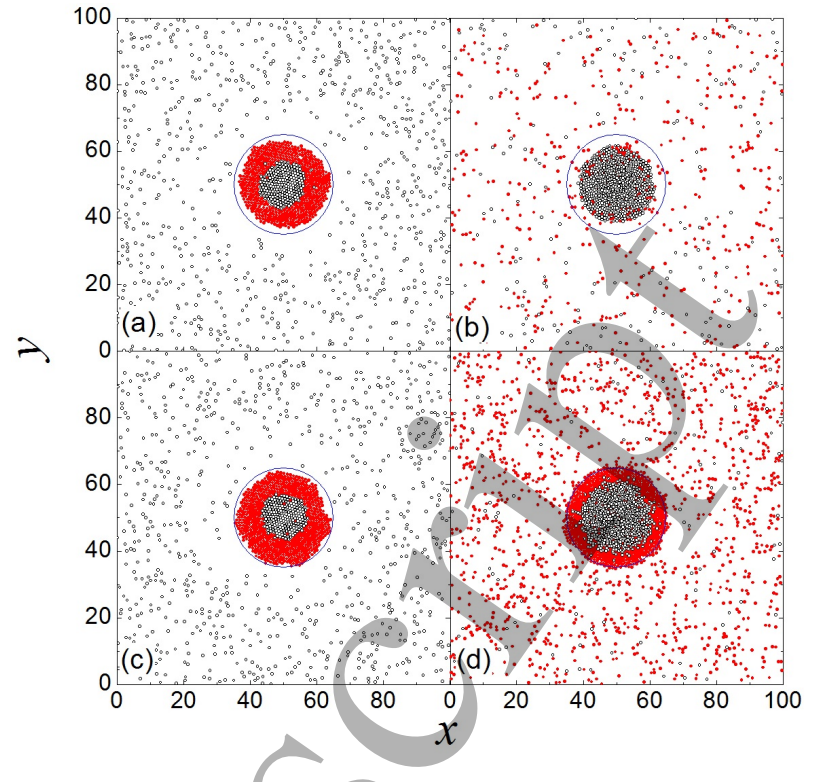

FIG. 3: (Color online). (a)-(d) Snapshots of the spatial configuration of a mixture with $N_{p}=1000$ (a) $N_{a}=500, v_{0}=1.0$; (b) $N_{a}=500, v_{0}=2.0$; (c) $N_{a}=600, v_{0}=1.0$; and (d) $N_{a}=2000, v_{0}=1.0$. All remaining simulation parameters are as in Fig. 1. The circles at the center of the simulation box delimit the parabolic trap.

here by traps

Simultaneously, as illustrated in Fig. 2(b), the number of dynamically trapped active particles, $N_{a}^{(t)}$, also increases with their total number, $N_{a}$. However, the dependence of the curves $N_{a}^{(t)}$ versus $N_{a}$ on $v_{0}$ is opposite to that observed for the passive mixture fraction: At a fixed concentration of Janus microswimmers, $N_{a}^{(t)}$ markedly decreases with increasing $v_{0}$. Therefore, the simulation data of Fig. 2(b) indicate that for the system parameters yielding the maximum trapping of the passive particles, i.e., $N_{a}=600$ and $v_{0}=2.0$, only about $15 \%$ to the active swimmers sit inside the trap.

The findings of Figs. 1 and 2 can be summarized as follows. A small fraction of active swimmers of relatively high self-propulsion speed ("hot" swimmers) added to a suspension of passive particles can drastically change the diffusive dynamics of the mixture overall. In particular, they can be used to confine the passive particles inside a designated storing area with efficiency that can be thus enhanced by a factor of up to four, or even larger.

Examples of the spatial distribution of active (red) and passive (black) particles in the simulation box at low temperature, $D_{T}=0.001$, are presented in Fig. 3. The snapshots of panels (a) and (c) illustrate situations when the trap apparently contains all active particles and only a small fraction of the passive ones. This occurrence is typical for low self-propulsion speeds; here, $v_{0}=1.0$. "Heating up" the system by raising $v_{0}$ from 1.0 up to 2.0 sharply changes the quasi-stationary mixture configuration: The initially "sleeping" active Janus swimmers 
leave the trap and "heat up" the passive component of the suspension. Under the stirring action of the Janus microswimmers, the passive particles then edge toward and eventually fall inside the trap in larger numbers, as shown in Fig. 3(b). On the contrary, the microswimmers themselves, due to their high motility, can easily escape from the trap. A simple force-balance argument yields the depinning condition $v_{0}>A r_{D}$, i.e., $v_{0}>1.5$ for the simulation parameters of Fig. 3. Of course, active particles can populate the area outside the trap also for lower self-propulsion speeds, but only at much higher concentrations, like in Fig. 3(d).

We recall that the above mechanism is a transient effect that works only for $\tau_{a} \ll \tau_{p}$. In our simulations such condition was ensured by taking a very small thermal diffusion, $D_{T}=0.001$, and a long simulation time, $t_{e}=6 \cdot 10^{4}$, so that $L^{2} \gg 4 D_{T} t_{e}$. At high temperatures, when the spatial diffusion induced by thermal fluctuations becomes appreciable, see Fig. 1(a), the above condition breaks down and the dynamical behaviour of active and passive species becomes indistinguishable (not shown). The latter occurrence represents an equilibrium condition clearly expected in the asymptotic time limit, but of lesser usage for practical applications.

\section{Mean square displacement}

The diffusive properties of the mixture constituents are characterized by their mean square displacement (MSD) defined as $\left\langle\Delta r^{2}(t)\right\rangle=\left\langle[r(t)-r(0)]^{2}(t)\right\rangle$. To analyze the diffusion of the active fraction, in Fig. 4 we plotted the MSD of the active Janus particles taken at $t=t_{e}$, $\left\langle\Delta r^{2}\left(t=t_{e}\right)\right\rangle \equiv\left\langle\Delta r^{2}\left(v_{0}\right)\right\rangle$, versus $v_{0}$ for different concentrations, $N_{a}$, and a fixed number of passive particles, $N_{p}=1000$. The ensemble average (respectively over $N_{a}=10,200,500,600$ and 1000 active particles) was further averaged over five independent simulation runs to obtain the rather smooth curves displayed in Fig. 4.

For relatively low concentrations, $N_{a}<600$, active Janus microswimmers with $v_{0}<A r_{D}$ are strongly confined by the trap, so that for $v_{0}=1.0$ to 1.2 we obtained $\left\langle\Delta r^{2}\right\rangle \simeq 0$. Increasing the self-propulsion speed across the range 1.4 to 1.6 causes a quite abrupt MSD jump, which we relate to the escape, or depinning of the Janus particles from the trap. Finally, for even higher self-propulsion speeds, $v_{0}=1.6$ to 2.0 , the MSD appears to linearly increase with $v_{0}$. We explain this effect by invoking the high rate of collisions between active and passive particles occurring outside the trap. In contrast to the above picture, at high active swimmer concentrations, e.g., $N_{a}=1000$, the MSD depinning jump tends to disappear. In this regime, the almost linear dependence of the MSD on $v_{0}$ is related to the observation that, due to their large concentration, the number of microswimmers diffusing outside the trap is appreciable even at low $v_{0}$ and surely increases with it. Vice versa, the depinning

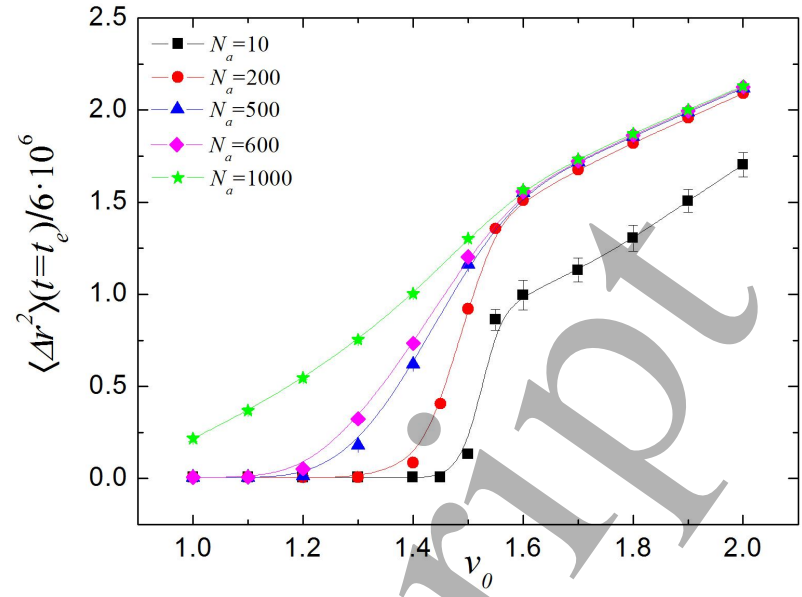

FIG. 4: (Color online). Mean square displacement, $\left\langle\Delta r^{2}\right\rangle$ for $t=t_{e}$, of active particles as a function of $v_{0}$, for systems with $N_{p}=1000$ and varying $N_{a}$ (see legends). Error bars are larger than the symbols only for $N_{a}=10$ and $v_{0}>1.5$. Interpolating curves are a guide to the eye. All remaining simulation parameters are as in Fig. 1.

step of the MSD curves becomes sharper and sharper as $N_{a}$ is lowered. Note that in Fig. 4 the step for $N_{a}=10$ occurs at $v_{0}=1.5$, as predicted.

\section{Detrapping of passive particles assisted by Janus microswimmers}

In Sec. III B we showed how active Janus particles can help store a large amount of passive particles in the trap. That result was achieved by injecting a small fraction of strongly self-propelled (or "hot") Janus swimmers in the passive suspension. This technique, in turn, raises a naturally related question that is especially meaningful in view of future applications: How can we remove the passive particles from the trap after they have accumulated there?

One simple method is suggested by inspecting the snapshots of the mixture configuration shown in Figs. 3(a,c): One can "cool down" the active component of the mixture, that is slow down the active Janus swimmers. To analyze this process in detail, we first calculated the number of trapped passive particles, $N_{p}^{(t)}$, versus the number of active particles, $N_{a}$, assuming that the former were initially distributed at random in space. The results are shown in Fig. 5(a) for different values of $v_{0}$. In contrast, to model detrapping all passive particles were initially placed inside the trap. As discussed at the top of Sec. III, the number of trapped passive particles, $N_{p}^{(t)}$, clearly depends on the initial conditions. Indeed, the detrapping rate of initially trapped passive particles will be lower than of randomly distributed passive particles. This hysteretic behavior is due to the fact that the simulation time, $t_{e}$, is much larger (shorter) than the re- 

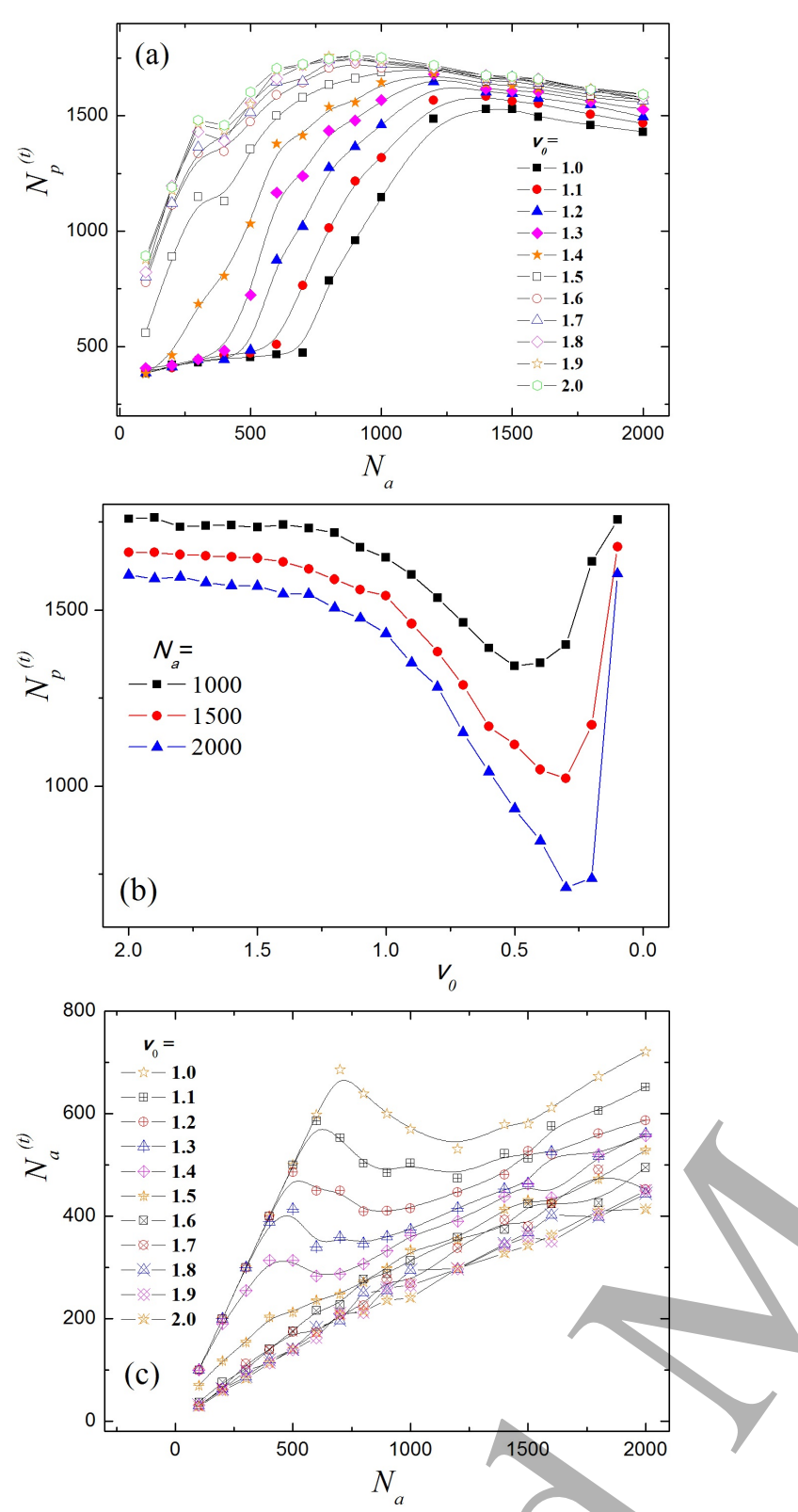

FIG. 5: (Color online). (a) Number of passive particles in the trap, $N_{p}^{(t)}$ versus total active particle number, $N_{a}$, for $N_{p}=2000$ and different $v_{0}$ (see legend). (b) Detrapping of passive particles (initially all placed in the trap): $N_{p}^{(t)}$ versus $v_{0}$ for $N_{p}=2000$ and different $N_{a}$ (see legend). (c) The number of active swimmers in the trap corresponding to (a). All the remaining simulation parameters are as in Fig. 1. Interpolating curves are a guide to the eye.

laxation time of the active (passive) particles. Therefore, all observables, like $N_{p}^{(t)}$, calculated over the run time $t_{e}$ are independent of the initial configurations of the active particles, but do depend, in general, on the initial distribution of the passive particles. Therefore, we chose three initial values of $N_{p}^{(t)}$ [resulting from the preparation procedure of Fig. 5(a), respectively, for $N_{a}=1000,1500$ and 2000] and then simulated the two species mixture by decreasing $v_{0}$ stepwise from the initial value 2.0 down to 0.1. Finally, Fig. 5(c) shows the number of trapped active swimmers corresponding to the situation shown in panel (a).

One notices immediately that $N_{p}^{(t)}$ in 5(a) exhibits a nonmonotonic dependence on $N_{a}$, which is consistent with the numerical data of Fig. 2(a). More remarkably, however, in Fig. 5(b) $N_{p}^{(t)}$ decreases monotonically with lowering $v_{0}$ from 2.0 down to around 0.5. A minimum trap occupancy is achieved for values of $v_{0}$ that grow lower as $N_{a}$ is raised. Therefore, by "cooling down" the active mixture component, we can actually extract a substantial fraction of passive particles stored in the trap.

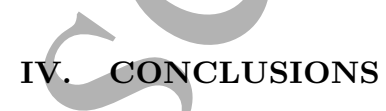

We have investigated the diffusive dynamics of a binary suspension of active Janus swimmers and passive colloidal particles in the presence of a local confining potential (trap). We have demonstrated the possibility of spatially manipulating the passive particles by means of the active swimmers with particular attention to their storing in and extraction from the trap. The former task was performed by injecting in the system a small fraction of active Janus swimmers with high self-propulsion speed ("hot" swimmers). The latter task, instead, was achieved by "cooling down" the active component of the mixture. In real experiments the speed of the self-propelled motion can be easily controlled, for instance, by regulating the intensity of the light illuminating the Janus microswimmers (thermo-phoresis) or the fuel concentration in the solution (chemo-phoresis).

\section{Acknowledgment}

This work was supported by the FWO-JSPS bilateral project, the "Odysseus" Program of the Flemish Government and the Flemish Research Foundation (FWO-Vl) (Belgium). W.Y. acknowledges the support from NSFC (No. 11204199), Shanxi Scholarship Council (No. 2016096), Fund for Shanxi Key Subjects Construction of China. F.N. is partially supported by the MURI Center for Dynamic Magneto-Optics via the AFOSR Award No. FA9550-14-1-0040, the Japan Society for the Promotion of Science (KAKENHI), the IMPACT program of JST, JSPS-RFBR Grant No. 17-52-50023, CREST Grant No. JPMJCR1676, the RIKEN-AIST Challenge Research Fund, and the Sir John Templeton Foundation. 
[1] J. Palacci, S. Sacanna, A. Vatchinsky, P. M. Chaikin, and D. J. Pine, J. Am. Chem. Soc., 2013, 135, 15978.

[2] N. Koumakis, A. Lepore, C. Maggi, and R. Di Leonardo, Nature Comm., 2013, 4, 2588.

[3] R. Dreyfus, J. Baudry, M. L. Roper, M. Fermigier, H. A. Stone, and J. Bibette, Nature, 2005, 437, 862

[4] G. A. Ozin,I. Manners, S. Fournier-Bidoz, and A. Arsenault, Adv. Mater., 2005, 17, 3011.

[5] J. Palacci, S. Sacanna, A. P. Steinberg, D. J. Pine, P. M. Chaikin, Science, 2013, 339, 936.

[6] I. Buttinoni, J. Bialke, F. Kummel, H. Lowen, C. Bechinger, and T. Speck, Phys. Rev. Lett., 2013, 110, 238301.

[7] W. Yang, V. R. Misko, K. Nelissen, M. Kong and F. M. Peeters, Soft Matter, 2012, 8, 5175.

[8] A. P. Petroff, X.-L. Wu, and A. Libchaber, Phys. Rev. Lett., 2015, 114, 158102.

[9] J. Dunkel, S. Heidenreich, K. Drescher, H. H. Wensink, M. Bar, and R. E. Goldstein, Phys. Rev. Lett., 2013, 110, 228102.

[10] E. Lauga and R. E. Goldstein, Phys. Today, 2012, 65, 30.

[11] K. Drescher, K. C. Leptos, I. Tuval, T. Ishikawa, T. J. Pedley, and R. E. Goldstein, Phys. Rev. Lett., 2009, 102, 168101.

[12] N. C. Darnton, L. Turner, S. Rojevsky, and H. C. Berg, J. Bacteriology, 2007, 189, 1756.

[13] J. Yan, M. Bloom, S. C. Bae, E. Luijten, and S. Granick, Nature, 2012, 491, 578.

[14] Y. Zhao, H. C. Shum, H. Chen, L. L. A. Adams, Z. Gu, and D. A. Weitz, J. Am. Chem. Soc., 2011, 133, 8790.

[15] H.-R. Jiang, N. Yoshinaga, and M. Sano, Phys. Rev. Lett., 2010, 105, 268302.

[16] S. Jiang, Q. Chen, M. Tripathy, E. Luijten, K. S. Schweizer, and S. Granick, Adv. Mater, 2010, 22, 1060

[17] C. Tang, C. Zhang, J. Liu, X. Qu, J. Li, and Z. Yang, Macromolecules, 2010, 43, 5114.

[18] P. K. Ghosh, V. R. Misko, F. Marchesoni, and F. Nori Phys. Rev. Lett., 2013, 110, 268301.
[19] H. L. Yu, A. Kopach, V. R. Misko, A. A. Vasylenko, D. Makarov, F. Marchesoni, F. Nori, L. Baraban, G. Cuniberti, Small, 2016, 12, 5882.

[20] J. J. Molina and R. Yamamoto, Molecular Physics, 2014, 112, 1389.

[21] C. Valeriani, M. Li, J. Novosel, J. Arlt and D. Marenduzzo, Soft Matter, 2011, 7, 5228.

[22] G. Gregoire, H. Chate, and Y. Tu, Phys. Rev. E, 2001, 64, 011902.

[23] X. Wu, and A. Libchaber, Phys. Rev. Lett., 2000, 84, 3017-3020.

[24] A. Morozov and D. Marenduzzo, Soft Matter, 2014, 10, 2748.

[25] T. V. Kasyap, D. L. Koch, and M. Wu, Phys. Flu., 2014, 26, 081901.

[26] S. A. Mallory, C. Valeriani, and A. Cacciuto, Phys. Rev. E, 2014, 90, 032309 .

[27] A. Kaiser and H. Lowen, J. Chem. Phys., 2014, 141, 044903.

[28] Y.-S. Su, H.-C. Wang, and L. I, Phys. Rev. E, 2015, 91, 030302(R).

[29] K.-A. Liu and L. I, Phys. Rev. E, 2013, 88, 033004.

[30] L. Angelani, C. Maggi, M. L. Bernardini, A. Rizzo, and R. Di Leonardo, Phys. Rev. Lett., 2011, 107, 138302.

[31] R. C. Krafnick and A. E. Garcia, Phys. Rev. E, 2015, 91, 022308.

[32] Y. Fily and M. Cristina Marchetti, Phys. Rev. Lett., 2012, 108,235702.

[33] W. Yang, V. R. Misko, J. Tempere, M. H. Kong, and F. M. Peeters, Phys. Rev. E, 2017, 95, 062602.

[34] R. Di Leonardo, S. Keen, F. Ianni, J. Leach, M. J. Padgett, an G. Ruocco, Phys. Rev. E, 2008, 78, 031406.

[35] X. Yang, C. Liu, Y. Li, F. Marchesoni, P. Hänggi, and H. P. Zhang, Proc. Natl. Acad. Sci. U.S.A., 2017, 114, 9565.

[36] T. Debnath, Y. Li, P. K. Ghosh, and F. Marchesoni, Phys. Rev. E, 2018, in press. 